\title{
Estimation of proton configurations from NOESY spectra
}

\author{
Michael Habeck*, Wolfgang Rieping* and Michael Nilges* \\ *Institut Pasteur, 25-28, rue du Dr. Roux, 75015 Paris, France
}

\begin{abstract}
Nuclear Overhauser effect spectroscopy (NOESY) data contain information about the geometry of a system of magnetically interacting nuclear spins. They thus allow to determine the positions of atoms in space and even suffice to infer the entire structure of a biomolecule. Yet, interpretation of NOESY spectra is often still qualitative. This is mostly due to the complexity of the theories that describe the data. We outline a Bayesian algorithm that estimates the configuration of protons by analysing unassigned NOESY volumes which may stem from spectra recorded at different mixing times. The method relies on the calculation of peak volumes with a relaxation matrix model and thereby incorporates spin-diffusion effects. Proton coordinates and nuisance parameters, such as the scales and errors of the spectra, are estimated using Markov Chain Monte Carlo sampling.
\end{abstract}

\section{INTRODUCTION}

If a system of nuclear spins is driven out of thermal equilibrium, dissipative interactions lead to a damping of the perturbed magnetisation such that it finally returns to its equilibrium value. The nuclear Overhauser effect (NOE) [1] is due to the phenomenon that nuclear spins do not relax independently but influence each other: Dipolar interactions transfer magnetisation through space and cause an increase or decrease in magnetisation depending on the distances to other spins. This is utilised in the NOESY experiment [2]. A sequence of radio-frequency pulses is applied to the system and creates a non-equilibrium distribution of nuclear spins which exchange longitudinal magnetisation through dipolar relaxation. Solomon's equations $[1,2]$ describe the evolution of the deviations $\Delta M_{z k}$ of longitudinal magnetisation for every spin $k=1, \ldots, N$ during a mixing period of length $\tau_{m}$ :

$$
\frac{\mathrm{d}}{\mathrm{d} t} \Delta \mathbf{M}_{z}(t)=-\mathbf{R} \Delta \mathbf{M}_{z}(t), \quad 0<t<\tau_{m} .
$$

In these rate equations the relaxation matrix $\mathbf{R}$ accounts for the return to the equilibrium magnetisation. Since the efficiency of magnetisation exchange depends on the internuclear distances $r_{k l}$, NOESY reveals geometrical information. The relaxation rates

$$
R_{k l}=\sigma\left(\tau_{c}\right) r_{k l}^{-6}, \quad R_{k k}=\rho\left(\tau_{c}\right) \sum_{l \neq k} r_{k l}^{-6}
$$

exhibit a typical $r^{-6}$ dependence stemming from the distance dependence of dipolar interactions. Non-zero cross-relaxation rates $R_{k l}$ are responsible for the ocurrence of 
cross-peaks in NOESY spectra. The auto-relaxation rates $R_{k k}$ balance the loss of magnetisation due to cross-relaxation; other relaxation mechanisms than dipolar relaxation are neglected in (2). The proportionality constants $\sigma\left(\tau_{c}\right)$ and $\rho\left(\tau_{c}\right)$ in (2) depend on the correlation time $\tau_{c}$ of molecular tumbling in solution. This random process is mainly responsible for relaxation. Integration of Solomon's equations yields the dependence of the volumes of the NOESY peaks on the mixing time $\tau_{m}[2]$ :

$$
\mathbf{V}\left(\tau_{m}\right)=\exp \left\{-\tau_{m} \mathbf{R}\right\} \mathbf{V}(0)=\mathbf{A}\left(\tau_{m}\right) \mathbf{V}(0)
$$

The equilibrium volumes $\mathbf{V}(0)=\gamma \mathbf{I}$ are diagonal, but their scale $\gamma$ is unknown. Calculation of the volumes involves evaluation of the matrix exponential

$$
\mathbf{A}(t)=\exp \{-t \mathbf{R}\}
$$

The mixing coefficients $A_{k l}(t)$ describe the build-up of NOESY cross-peaks: The transfer efficiency first grows with increasing mixing time, reaches a maximum and finally decays exponentially.

Structure determination from NOESY spectra [3] involves two steps: First, crosspeaks are assigned to pairs of protons via their chemical shifts. Second, inter-proton distances are derived from peak volumes and used as constraints in an optimisation of the structure. In the following, we will skip the assignment step and try to infer the coordinates of unidentified protons directly from the peak volumes. We reduce the macromolecular structure to those protons that can be seen in the NOESY experiment. We would need to identify them, if we wanted to include information about the covalent topology.

\section{PROBABILISTIC MODELLING}

We consider $N$ protons which spins interact via (1) and infer their positions $\mathbf{x}_{k}$ from unassigned NOESY volumes $D=\left\{V_{k l},(k, l) \in I\right\}$. The index list $I$ does not include all possible pairs since usually diagonal peaks cannot be resolved and some cross-peaks will be undistinguishable from noise. The likelihood resulting from NOESY data recorded at mixing time $\tau$ is

$$
L(\mathbf{X}, \gamma, \sigma)=\sigma^{-n} \exp \left\{-\frac{1}{2 \sigma^{2}} \chi^{2}(\mathbf{X}, \gamma)\right\} .
$$

Use a of Lognormal model to describe observation of the positive volumes involves the misfit:

$$
\chi^{2}(\mathbf{X}, \gamma)=\sum_{(k, l) \in I} \log ^{2}\left(\gamma A_{k l}(\tau) / V_{k l}\right)
$$

The hypothesis parameters are the three-dimensional coordinates $\mathbf{X}=\left(\mathbf{x}_{1}, \ldots, \mathbf{x}_{N}\right)^{T}$ used to predict the mixing coefficients $A_{k l}(\tau)$ (Eq. 4), a scale $\gamma$ that calibrates the equilibrium volumes in (3), and the error $\sigma$. We assume a Boltzmann distribution as conformational prior: the protons make up a gas at temperature $\beta^{-1}$ with potential 

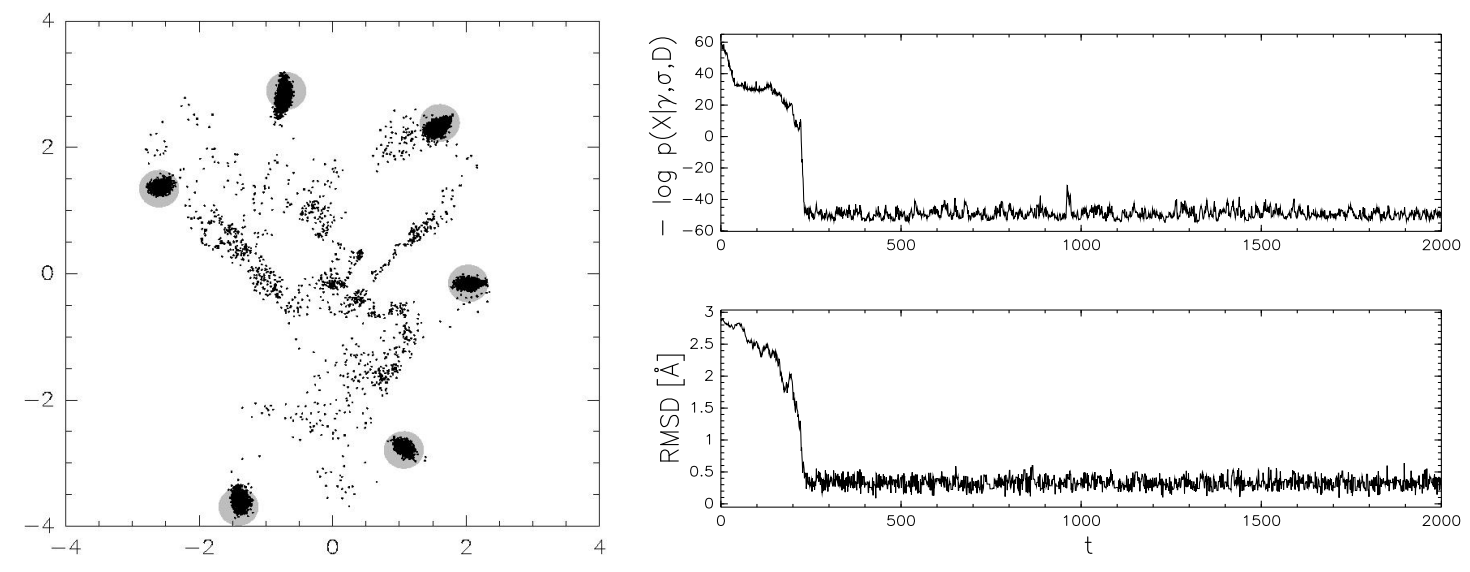

FIGURE 1. Left: conformational samples shown as black dots, big grey circles indicate the positions of the protons in the tryptophan ring. Right: traces of the negative logarithm of the posterior and of the RMS deviation between true and estimated coordinates.

energy $E(\mathbf{X})$ penalizing interpenetration of protons. Furthermore, we use Jeffreys' prior for the nuisance parameters $\gamma$ and $\sigma$. Bayes' theorem yields the posterior

$$
p(\mathbf{X}, \gamma, \sigma \mid D) \propto \sigma^{-(n+1)} \gamma^{-1} \exp \left\{-\frac{1}{2 \sigma^{2}} \chi^{2}(\mathbf{X}, \gamma)-\beta E(\mathbf{X})\right\}
$$

and allows us to invert the noisy and incomplete volume matrix by fitting the proton positions directly to the data. Our approach is in spirit similar to the CLOUDS algorithm [4]. There, a proton gas is condensed such as to fulfil distances that stem from reconstructed relaxation rates. However, we do not separate estimation of relaxation rates from fitting of proton coordinates. Using equation (2), we parametrise the relaxation rates directly in proton coordinates.

\section{POSTERIOR SAMPLING}

We use Gibbs sampling [5] to simulate the joint posterior $p(\mathbf{X}, \gamma, \sigma \mid D)$. Random number generators for the Gamma and the Lognormal distribution generate samples from the conditional posteriors $p(\sigma \mid \mathbf{X}, \gamma, D)$ and $p(\gamma \mid \mathbf{X}, \sigma, D)$, respectively. Simulation of the conformational conditional posterior

$$
p(\mathbf{X} \mid \gamma, \sigma, D) \propto \exp \left\{-\frac{1}{2 \sigma^{2}} \chi^{2}(\mathbf{X}, \gamma)-\beta E(\mathbf{X})\right\}
$$

is more involved. $p(\mathbf{X} \mid \gamma, \sigma, D)$ is a modified Boltzmann distribution in which the "forces" resulting from $\chi^{2}$ counteract the repulsive terms in the potential energy $E$. Hybrid Monte Carlo [6] distributes the protons in space such that the peak volumes are reproduced best while maintaining a low potential energy. 

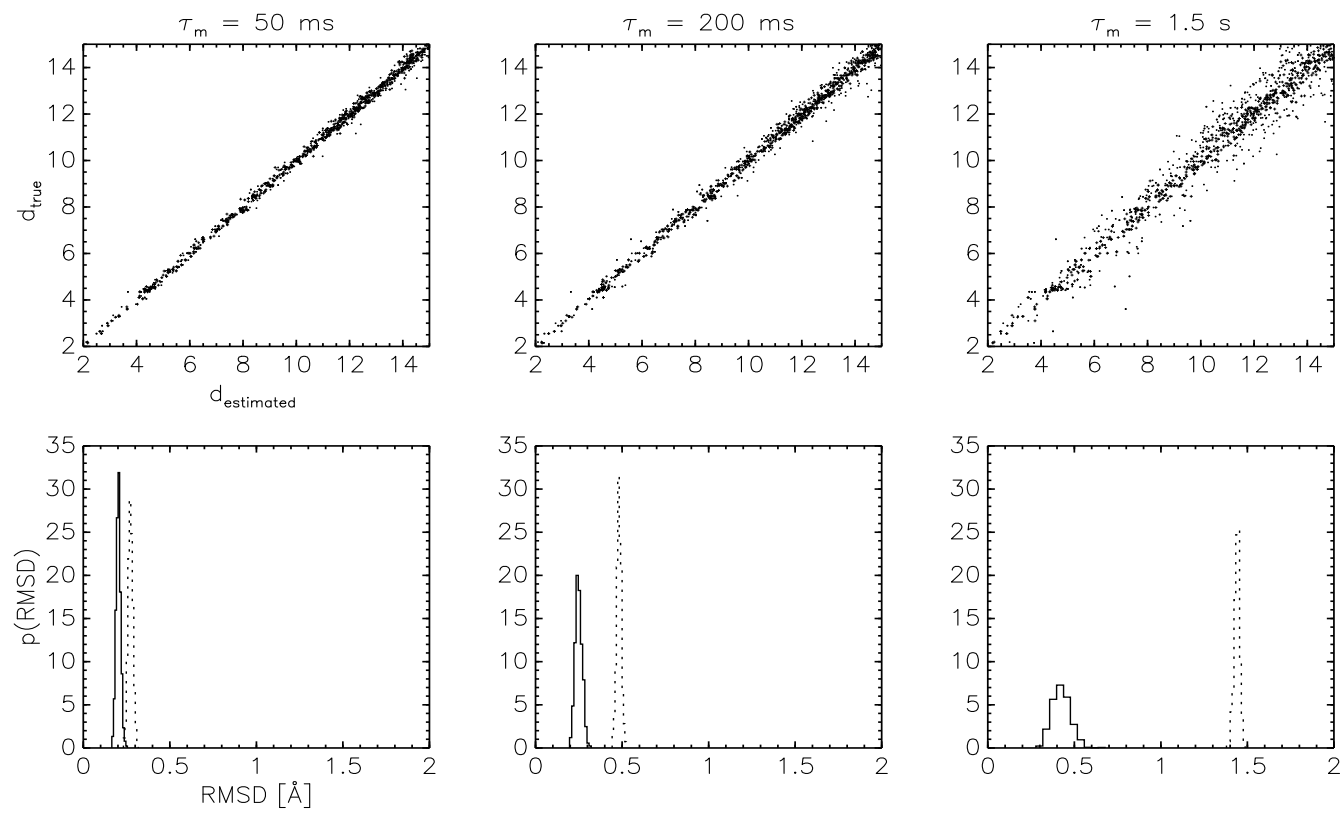

FIGURE 2. In the upper row the estimated distances are plotted against the true distances using the ISPA model. The bottom row shows histograms of the RMSD to the true structure. The dashed histograms are the results of the ISPA based analysis, the solid histograms are calculated on grounds of the relaxation matrix model.

\section{APPLICATIONS}

In the following applications we analysed artificial data assuming a rotational correlation time of $\tau_{c}=8.5 \mathrm{~ns}$ and a spectrometer frequency of $600 \mathrm{MHz}$. We first applied the algorithm to a simple system: The side chain of the amino acid tryptophan is made up of two rings with six protons that can exchange magnetisation during the NOESY experiment. We simulated data at a mixing time of $100 \mathrm{~ms}$ with an error of $10 \%$. It was assumed that diagonal peaks could not be resolved; furthermore, volumes below 0.001 were considered to be too weak to be observable. In this example, Hybrid Monte Carlo is able to find the mode of the posterior distribution. After a burn-in period, conformational samples are generated that scatter around the true positions of the protons (Fig. 1). The negative logarithm of the posterior indicates the end of the burn-in by a sudden jump to a minimum value. In general, however, this criterion is not sufficient to guarantee convergence of the Markov chain. In more complex problems it is likely that the Markov chain gets stuck in one of the posterior modes. We should then use the replica scheme outlined in [7].

Throughout the applications that follow we will use a more complex model system (the same one as in [7]): We assume that we measure magnetisation exchange between the amide protons of the Fyn SH3 domain. Such data can be measured by performing NOESY experiments on a deuterated sample [8]. We have to estimate the coordinates of 55 protons. 

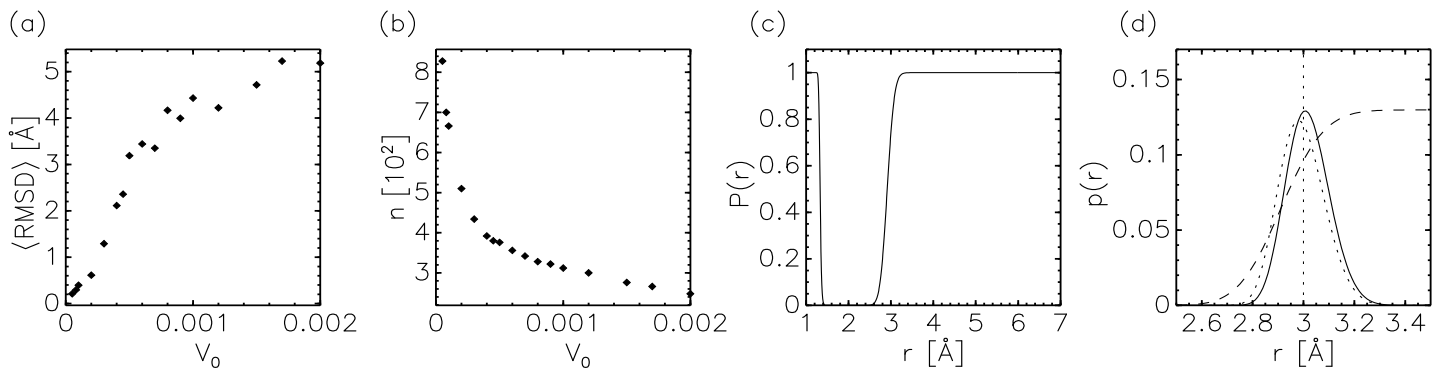

FIGURE 3. a: average RMSD against noise threshold. b: dependence of the number of observable volumes on the noise threshold $V_{0}$. c: likelihood factor resulting from modelling non-observed volumes. d: likelihood factors from observed (dotted line) and non-observed volumes (dashed) line. The total likelihood is the product of the two factors (solid line).

\section{Comparison with the isolated spin pair approximation}

For short mixing times, the matrix exponential (4) can be approximated by a first order expansion leading to the isolated spin pair approximation (ISPA):

$$
V_{k l} \propto r_{k l}^{-6}, \quad k \neq l .
$$

The ISPA is almost always used in structure calculation from NOESY data. This is due to the computational overload of a full treatment involving calculation of the relaxation matrix and its exponential. In order to measure data where this approximation is still appropriate, mixing times are usually kept short (initial rate regime). This has the disadvantage that one can only measure data for distances of protons that are very close in space. Futhermore, the signal-to-noise ratio is unfavourable in the initial rate regime. We drew conformational samples for data generated at three different mixing times $\tau_{i}=$ $50 \mathrm{~ms}, 200 \mathrm{~ms}$ and $1.5 \mathrm{~s}$ (again $\tau_{c}=8.5 \mathrm{~ns}$ ). The error was chosen to be $25 \%$. For the shortest mixing time, results of the relaxation matrix treatment and the ISPA-based approach are virtually identical (see Fig. 2). The correlation between the true distances and their estimated values is quite high. This equivalence is gradually destroyed at longer mixing times. Structures calculated on the basis of the ISPA get worse, whereas the RMSD values of the relaxation matrix approach are only slightly shifted towards larger values.

\section{Incomplete volume matrices}

Cross-peaks are only observable if their strength exceeds a certain noise threshold $V_{0}$. In order to investigate the influence of such a threshold on the quality of the coordinates, we generated data with different $V_{0}$ values for a fixed mixing time of $100 \mathrm{~ms}$ and a $10 \%$ error. With increasing threshold value, we can observe fewer cross-peaks. The number of observable volumes roughly follows the power law $n \propto V_{0}^{-1 / 3}$. As a result, the structures get worse with increasing threshold $V_{0}$ (see Fig. $3 \mathrm{a}$ and $3 \mathrm{~b}$ ). We can include the information that a peak has not been observed. The probability for a cross-peak to 
(a)

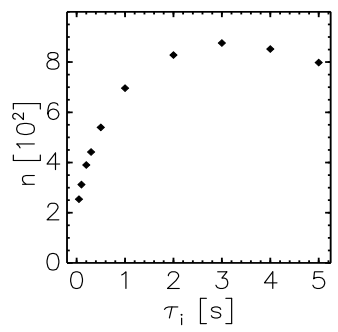

(b)

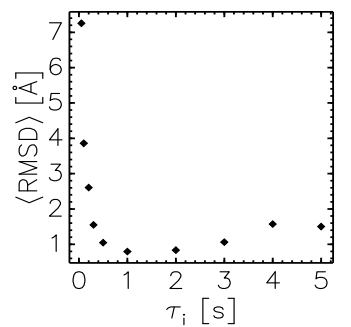

(c)

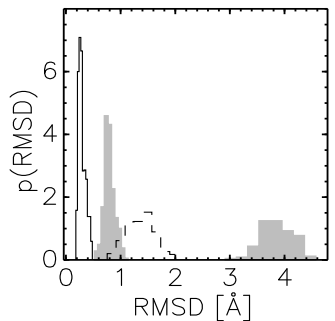

(d)

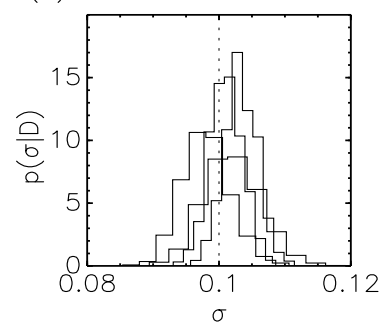

FIGURE 4. a: number of observed volumes against mixing time. b: average RMSD against mixing time when analysing each data set independently. c: RMSD histograms for a bad $\left(\tau_{m}=100 \mathrm{~ms}\right)$ and the best analysis $\left(\tau_{m}=1 \mathrm{~s}\right)$ of a single data set are shaded. The dashed RMSD histogram is obtained from a simultaneous analysis of all data sets with errors fixed to their true values. The solid histogram stems from a simulation where the errors are also estimated. d: posterior distributions of individual errors $\sigma_{i}$ estimated through Gibbs sampling. The dotted vertical line indicates the true value.

be smaller than $V_{0}$ is

$$
P\left(V_{k l}<V_{0} \mid \mathbf{X}, \gamma, \sigma\right)=\frac{1}{2}\left(1+\operatorname{erf}\left(\log \left(\gamma A_{k l}(\tau) / V_{0}\right) / \sqrt{2 \sigma^{2}}\right)\right) .
$$

The resulting factor in the likelihood penalizes distances that are too small. This counteracts the effect that configurations become too compact because observations are missing. Inclusion of non-observed cross-peaks shifts the posterior distribution towards the correct value (Fig. 3c,d).

\section{Analysis of multiple spectra}

NOESY spectra are often recorded at different mixing times. Traditional approaches encounter difficulties when analysing multiple spectra at the same time. They have to resort to workarounds where, for example, each spectrum is analysed separately and relaxation rates are averaged (see [9]). In a Bayesian analysis, combined use of multiple spectra is straightforward: the likelihood is just a product of factors (5); each factor involves only volumes that have been recorded at the same mixing time. We generated spectra for ten different mixing times with a $10 \%$ error and a sensitivity threshold of 0.001 . We first analysed the spectra individually. We note that with increasing mixing time, we can measure more volumes if the threshold value is considered fixed (Fig. 4a). The number of measurable volumes reaches a maximum and decreases again for mixing times longer than $3 \mathrm{~s}$. The accuracy of the estimated coordinates improves with increasing mixing time since more measurements are available (Fig. 4b). We also analysed all spectra at the same time with the errors fixed to their true values. The structures are less accurate than those obtained from the best data set $\left(\tau_{m}=1 \mathrm{~s}\right)$. The protons are pulled to less accurate configurations by data sets that result in worse structures $\left(\tau_{m}=50\right.$ or $\left.100 \mathrm{~ms}\right)$. Only when estimating the errors of the spectra, the structures get better than in the calculations based on single data sets (Fig. 4c). Each spectrum is then weighted according to its quality and its consistency with the other 


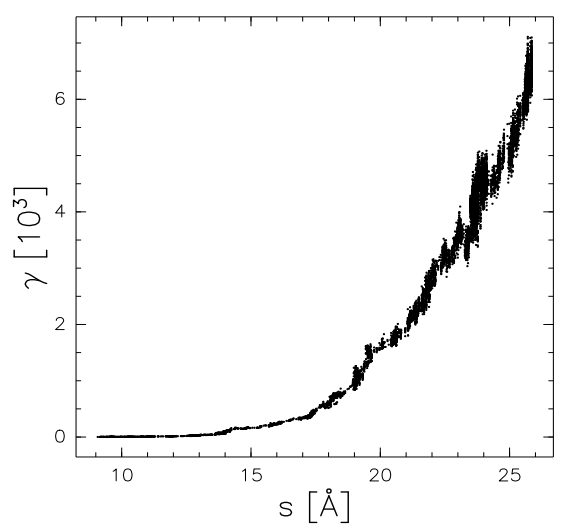

FIGURE 5. Estimated spectral scale against the radius of gyration $s$ of the generated configurations. The samples approximately follow the law $s \propto \gamma^{6}$, as expected from the invariance (11).

data. We do not need to specify the weights beforehand, but estimate them along with the coordinates (Fig. 4d).

\section{Estimation of nuisance parameters}

Estimation of spectrum errors is straightforward: posterior samples are generated from a Gamma distribution. If volumes are modelled that have not been observed, the conditional posterior is no longer a known distribution. Thus, estimation becomes more complicated but is still feasible. In constrast, estimation of the spectral scale is problematic due to the following quasi-invariance: An overall scaling of the configuration roughly leads to a scaling of the NOESY volumes:

$$
\mathbf{x}_{i} \rightarrow \alpha \mathbf{x}_{i} \quad \Rightarrow \quad R_{k l} \rightarrow \alpha^{-6} R_{k l} \quad \Rightarrow \quad V_{k l} \rightarrow \alpha^{-6} V_{k l},
$$

where the transformation of volumes is only approximately valid, but will become better for large $\alpha$. This scaling can be compensated by the factor $\gamma$. Thus, nothing will stop the algorithm from blowing up the structures while adjusting $\gamma$ (Fig. 5). In order to prevent this, we need to introduce a prior term that confines the protons to a finite volume. Structures of folded macromolecules are globular and the distances between protons fall into a finite range. Such knowledge should be included in the prior; a possibility would be a radius of gyration term or an approximate proton density derived from known biomolecular structures.

\section{CONCLUSIONS}

Probability theory is well suited for the analysis of NOESY spectra. We do not need to assign the resonances: The proton coordinates are directly estimated from noisy and incomplete volume matrices using Markov Chain Monte Carlo sampling. Posterior simulation generates an ensemble of proton configurations that resembles the true structure. An 
open problem is to embed a complete macromolecular structure into the proton gas. By using a relaxation matrix model, we can account for spin diffusion. Thus, the approach is superior to an analysis based on the ISPA. We are always dealing with incomplete data. We can improve the analysis by modelling volumes that are not observed because the cross-peak is too weak (Eq. (10)) which leads to something similar to an "anti-distance restraint" $[10,11]$. Combined analysis of multiple spectra is straightforward and becomes powerful when estimating the spectrum errors along with the coordinates. In real applications one has to estimate the spectral scale. To this end, one must break the quasiinvariance (11). This could be accomplished by extending the conformational prior. One could, for example, use a radius of gyration term (Gaussian prior) to confine the proton gas a to a spherical volume. So far, the algorithm has only been tested on simulated data. The method still needs to prove useful for analysing real data.

\section{ACKNOWLEDGMENTS}

The work was supported by the EU grant QLG2-CT-2000-01313.

\section{REFERENCES}

1. Solomon, I., Phys. Rev., 99, 559-565 (1955).

2. Macura, S., and Ernst, R. R., Molecular Physics, 41, 95-117 (1980).

3. Wüthrich, K., NMR of Proteins and Nucleic Acids, John Wiley, New York, 1986.

4. Grishaev, A., and Llinás, M., Proc. Natl. Acad. Sci. USA, 99, 6707-6712 (2002).

5. Geman, S., and Geman, D., IEEE Trans. PAMI, 6, 721-741 (1984).

6. Duane, S., Kennedy, A. D., Pendleton, B., and Roweth, D., Phys. Rev. Lett. B, 195, 216-222 (1987).

7. Habeck, M., Rieping, W., and Nilges, M., "A new principle for macromolecular structure determination," in 23rd International Workshop on Bayesian Inference and Maximum Entropy Methods in Science and Engineering, edited by G. Erickson and Y. Zhai, American Institute of Physics, 2004, pp. 157-166.

8. Mal, T. K., Matthews, S. J., Kovacs, H., Campbell, I. D., and Boyd, J., J. Biomol. NMR, 12, 259-276 (1998).

9. Boelens, R., Koning, T. M. G., and Kaptein, R., J. Mol. Struct., 173, 299-311 (1989).

10. De Vlieg, R., Boelens, R., Scheek, R. M., Kaptein, R., and van Gunsteren, W. F., Isr. J. Chem., 27, 181-186 (1986).

11. Brüschweiler, R., Blackledge, M., and Ernst, R. R., J. Biomol. NMR, 1, 3-11 (1991). 